\title{
Análisis prospectivo del mercado chino para la etapa post COVID del sector turístico cubano
}

\section{Prospective analysis of the Chinese market for the post Covid stage of the}

\section{Cuban tourism sector}

$1 \quad$ Melissa Lemes Reyes

Universidad de La Habana, Facultad de Turismo, Licenciatura en Turismo. La Habana, Cuba

1melylr2001@nauta.cu

2 Claudia Domínguez Cantera

Universidad de La Habana, Facultad de Turismo, Licenciatura en Turismo. La Habana, Cuba

claudiacantera@nauta.cu

3 Yasser Vázquez Alfonso

Universidad de La Habana, Facultad de Turismo, Departamento de Turismo. La Habana, Cuba

yalfos1@gmail.com

4 Luis Efrain Velastegui Lopez $\quad$ (iD) https://orcid.org0000-0002-7353-5853

Universidad Técnica de Babahoyo, Babahoyo, Ecuador

evelasteguil@utb.edu.ec

Artículo de Investigación Científica y Tecnológica

Enviado: 04/12/2021

Revisado: $16 / 12 / 2021$

Aceptado: 23/01/2022

Publicado:26/02/2022

DOI: https://doi.org/10.33262/exploradordigital.v6i1.2077

Cítese: $\quad$ Lemes Reyes, M., Domínguez Cantera, C., Vázquez Alfonso, Y., \& Velastegui Lopez, L. E. (2022). Análisis prospectivo del mercado chino para la etapa post COVID del sector $\begin{array}{llll}\text { turístico cubano. } & \text { Explorador } & \text { Digital, } & \text { 6(1), }\end{array}$ https://doi.org/10.33262/exploradordigital.v6i1.2077

EXPLORADOR DIGITAL, es una Revista electrónica, Trimestral, que se publicará en soporte electrónico tiene como misión contribuir a la formación de profesionales competentes con visión humanística y crítica que sean capaces de exponer sus resultados investigativos y científicos en la misma medida que se promueva mediante su intervención cambios positivos en la sociedad. https://exploradordigital.org

La revista es editada por la Editorial Ciencia Digital (Editorial de prestigio registrada en la Cámara Ecuatoriana de Libro con No de Afiliación 663) www.celibro.org.ec 


\section{Palabras}

claves:

escenarios

prospectivos, mercado chino, sector turístico.

\section{Keywords:}

prospective scenarios,

Chinese

market, Cuban tourist sector.

\section{Resumen}

Esta investigación constituye un estudio de las perspectivas del mercado chino en la etapa post Covid del sector turístico cubano y su objetivo fue construir los escenarios prospectivos posibles y probables para su comportamiento. Para ello se ejecutó un análisis respaldado en información primaria y se emplearon técnicas cualitativas y cuantitativas de recogida de información tales como reuniones virtuales y encuestas a especialistas y expertos. Como parte de la investigación se empleó una metodología compuesta por herramientas y técnicas las cuales son: tormenta de ideas, el análisis DAFO (Debilidades, Amenazas, Fortalezas y Oportunidades), el análisis estructural, el análisis morfológico y un análisis probabilístico. Las principales herramientas utilizadas se apoyaron en los softwares siguientes: MICMAC (Matriz de Impactos Cruzados Multiplicación Aplicada a una Clasificación), MORPHOL (Morfológico), Smic-Prob-Expert (Sistema de Matrices de Impactos Cruzados) y el MULTIPOL (MULTI-criterio y Política). Como resultado se obtuvo un análisis del comportamiento futuro del mercado chino y se determinó los escenarios prospectivos con más probabilidad de ocurrencia o referencia; mediante los cuales directivos del MINTUR tomarán decisiones con un mínimo de incertidumbre.

\section{Abstract}

This investigation constitutes a study of the perspectives of the Chinese market in the stage post Covid of the Cuban tourist sector and its objective was to build the possible and probable prospective scenarios for the behavior of the Chinese market. For it was executed it an analysis supported in primary information, and they were used technical qualitative and quantitative of such collection of information as meetings and surveys to specialists and experts. As part of the investigation, a methodology was used for the prospective scenario construction composed by tools and technical, which are storm of ideas, the analysis DAFO (Weaknesses, Threats, Strengths and Opportunities), the structural analysis, the morphological analysis and a probabilistic analysis. The main used tools leaned on in the following software's: MICMAC (Womb of Impacts Crossed Applied Multiplication to a Classification), MORPHOL (Morphological), Smic-Prob -Expert (System of Wombs of Crossed Impacts) and the MULTIPOL (Multi-approach 
and Politics). as a result, an analysis of the future behavior of the Chinese market was obtained and it was determined the prospective scenarios with more occurrence probability or referential; by means of which the directive of the MINTUR will take decisions about the perspectives of the Chinese market in the stage post-covid with a minimum of uncertainty.

\section{Introducción}

La tendencia en el sector turístico señala que habrá menos viajes, por lo que es necesario trazar y planificar nuevas acciones y estrategias de experiencias turísticas alineadas a la realidad post pandemia.

La prospectiva es un proceso sistemático y participativo desarrollado con el objetivo de generar conocimiento, "desarrollar inteligencia", sobre el futuro de la ciencia y la tecnología mediante la construcción de visiones a medio y largo plazo que permitan tomar decisiones hoy. Para ello, analiza los desarrollos de la ciencia, la tecnología, la sociedad y la economía junto con las interacciones que existen para poder identificar cuáles son las acciones y estrategias que es preciso poner en marcha para poder generar el mayor beneficio social, la situación futura más favorable (Cornejo et al., 2019).

Sánchez (2003) plantea que serán decisivos los factores institucionales de cómo van a reaccionar los gobiernos para impulsar la actividad turística con respecto al comportamiento del turista, el cual forzaría cambios cualitativos para adaptar la oferta turística a las nuevas preferencias. Es por eso por lo que los gestores de destinos turísticos tendrán que asumir la gestión integral de la demanda turística. Entre los principales países que han marcado tendencia en el turismo emisor se encuentra China. Los numerosos turistas chinos que pasan sus vacaciones más allá de sus fronteras han despertado un entusiasmo turístico sin precedentes.

Problema de investigación: Cuba, es un mercado emergente con posibilidades de potenciarse sus arribos, pero en la actualidad no se han realizado estudios desde el MINTUR sobre cómo puede comportarse el mercado chino.

Objetivo general: Definir los posibles escenarios para el estudio del comportamiento del mercado chino en la etapa post Covid del sector turístico cubano y que permita una mejora en la toma de decisiones por parte de los gestores del turismo en el destino Cuba. 
Objetivos específicos:

- Identificar las potencialidades y restricciones fundamentales del comportamiento del mercado emisor chino para la etapa post COVID del sector turístico cubano.

- Determinar las variables que más influyen en el comportamiento del mercador emisor chino.

Hipótesis: La definición de los posibles escenarios para el estudio del comportamiento del mercado chino en la etapa post COVID del sector turístico cubano permitirá una mejora en la toma de decisiones por parte de los gestores del turismo en el destino Cuba.

\section{Metodología}

La investigación realizada en el presente estudio es exploratoria. El diseño desarrollado se clasifica de tipo no experimental, ya que se estudiaron los fenómenos tal y como se dan en su contexto natural, para su posterior análisis sin una manipulación previa de las variables. Como métodos de investigación se utilizó el teórico, empírico y el estadísticomatemático.

Entre los teóricos se utilizaron: histórico-lógico para conocer el comportamiento y evolución de las diferentes posiciones con respecto a la aplicación del análisis prospectivo en el sector turístico, análisis-síntesis para llegar a conclusiones sobre la base de diferentes fuentes.

Como método empírico se emplearon: consulta de documentos para la revisión de la literatura especializada en la temática de análisis prospectivo, encuesta para obtener información sobre los criterios de los turistas chinos, consulta a experto y especialista para obtener información a partir de la experiencia de expertos y especialista en estudio de mercado turísticos.

Para el análisis de la información se usaron los métodos estadísticos-matemáticos mediante la estadística descriptiva para el procesamiento de la información a través de tablas, gráficos y estadígrafos, también se utilizaron los métodos de análisis prospectivos como el análisis estructural, el pág. 1análisis morfológico, el Sistema matricial de impactos cruzados

El análisis estructural es una herramienta que posibilita describir un sistema usando una matriz que evidencia todas las relaciones entre las variables que lo constituyen (Astigarraga, 2010). Según Godet (1993) y Delgado (2015), ayuda a identificar las variables que ejercen mayor influencia y aquellas más susceptibles de ser influenciadas dentro de un sistema. Se descompone en tres fases según Godet (2000) y Munera (2016): identificación de variables, descripción de relaciones entre variables y búsqueda de las variables clave a través del software MICMAC. 
La técnica de impactos cruzados apoya a la toma de decisiones y sus usos más frecuentes son valoración de alternativas tecnológicas, planeación estratégica de negocios y análisis de políticas. Consiste en explorar el futuro, de acuerdo con la ocurrencia o no, de una serie de eventos, dentro de un periodo de tiempo determinado. Sus fases según Sánchez (2003) son: definición del sistema, selección de los participantes, definición de eventos analizar, asignación de probabilidades de ocurrencia, construcción de la matriz de impactos cruzados, valoración impactos, cálculo de las probabilidades y análisis de sensibilidad.

El método Sistema matricial de impacto cruzado tiene como objetivo obtener un escenario deseable que será la base del modelo de simulación. Está constituido por un conjunto de hipótesis que podrían materializarse dentro de un periodo de tiempo. Esto significa que "se tendrán tantos escenarios posibles (imágenes finales), como combinaciones de juegos de hipótesis existan" (Cely, 1999). La ventaja que ofrecen los métodos de impacto cruzado es que estiman la probabilidad de ocurrencia de los diferentes eventos, y de las interrelaciones existentes entre ellos (Rincón \& Mujica, 2004).

El Análisis Morfológico (AM), según Munera (2016), es una herramienta que permite explorar la "forma" o "estructura" de un problema o sistema, descomponiéndolo en cada una de las variables que lo conforman, permitiendo identificar para cada variable o cuestión clave, una serie de hipótesis sobre su comportamiento futuro. Según (Astigarraga, 2010), explora de manera sistemática los futuros posibles a partir del estudio de todas las combinaciones resultantes de la descomposición de un sistema.

El método MULTIPOL es una herramienta prospectiva para orientar la toma de decisiones cuando se cuenta con múltiples opciones, y se debe identificar la más conveniente de acuerdo con el contexto. Su principal objetivo es contrastar diferentes acciones y soluciones, a partir de una matriz de análisis comparativo, la cual permitirá identificar los criterios y políticas alternativos que dan solución a los problemas de decisión.

\section{Resultados}

Como resultado del procesamiento de la matriz DAFO (ver figura 1), se obtuvo que el cuadrante de mayor valor se encuentra en la zona de incertidumbre, de esta manera los directivos del sector turístico cubano deben adoptar una estrategia adaptativa que minimice las debilidades y maximice las oportunidades para atraer al mercado chino en la etapa post COVID. De este resultado que se deben potenciar productos turísticos diversificados, productos turísticos SemiFIT, productos turísticos privados para grupos, Tour para conocer y disfrutar las experiencias y las comunidades locales, Networking, utilización de tarjeta de crédito, uso de las plataformas Ctrip, Tuniu.com, Qunar.com, 
LY.com, Fliggy.com, uso de redes sociales, utilización de sistema de reserva online y de Wechat, Weibo, FeiZhu, Tuniu, Ctrip, BaiduDitu.

Figura 1

\section{Procesamiento de la matriz DAFO}

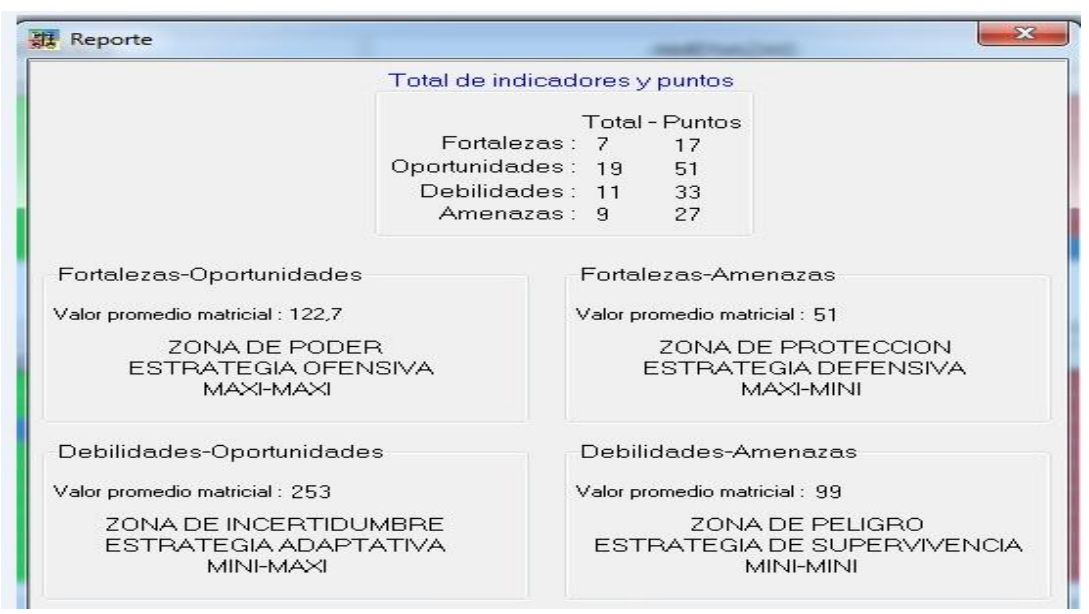

Análisis de las encuestas a turistas chinos

Con el objetivo de describir el comportamiento de las diferentes variables utilizadas en la encuesta realizada a los turistas chinos, se aplica un análisis estructural utilizando el software MICMAC para identificar las variables influyentes y dependientes en el estudio. En la figura 2 se muestra el gráfico del plano de influencia de las variables analizadas en la encuesta y se observa que las variables NVA(Número de visitas anteriores), EPVA (Experiencia personal de viajes anteriores), VMLH (Visita a monumentos y lugares históricos), ACRE (Almorzar o cenar en restaurantes emblemáticos de la ciudad) están en la zona de poder y se clasifican como variables explicativas del sistema, su progreso determina la orientación y el impulso para potenciar el mercado chino en el sector turístico cubano. La variable Principal motivo de viaje (PMV)y Zona turística (Z) se encuentran en la zona de conflicto y se clasifican en variables de enlaces, estas al igual que las primeras tienen una importancia en el estudio y comportamiento del mercado chino en la etapa post COVID y poseen el mayor número de relaciones con el resto de los elementos del sistema. Algunas de esas variables coinciden con los criterios dados por Mao \& Zhang (2014) y Lojo (2016), comentan que para los estudios del mercado emisor chino es necesario analizar las motivaciones, elección del destino y la experiencia en el lugar. 


\section{Figura 2}

Identificación de variables importantes según los criterios de turistas chinos Potential direct influence/dependence map

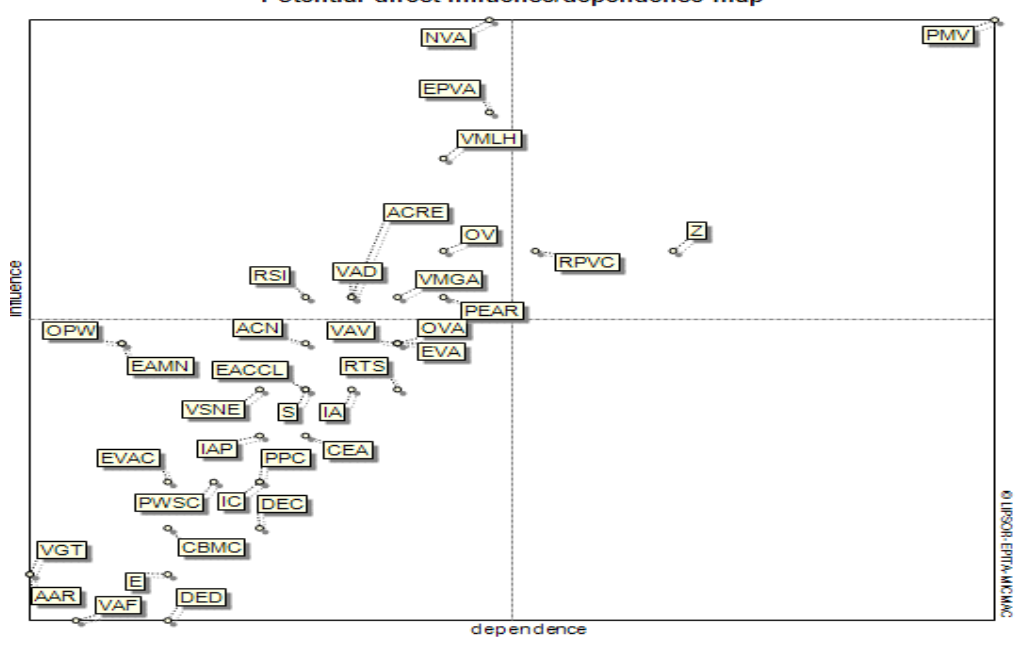

Para el estudio de las variables que más influyen en los criterios de los turistas chinos se realizó el gráfico de influencias directas potenciales donde se visualiza las relaciones de influencias relativamente importantes y relaciones de influencias más importantes (ver figura 3), destacándose las variables Experiencia personal de viajes anteriores (EPVA), Realiza su primer viaje a Cuba (RPVC), Número de visitas anteriores (NVA), Estancia en la visita anterior más reciente (EVA), Organización del actual viaje (OVA), Otra vía (OV), Principal motivo de viaje (PMV), Participar en eventos académicos o reuniones (PEAR) y Otras páginas webs (OPW). Estas variables se consideran estratégicas y son la base para estudios sobre el comportamiento del mercado chino en el sector turístico cubano y coinciden con lo planteado por Hernández \& Machado (2010) en su estudio realizado.

\section{Figura 3}

Influencias directas potenciales

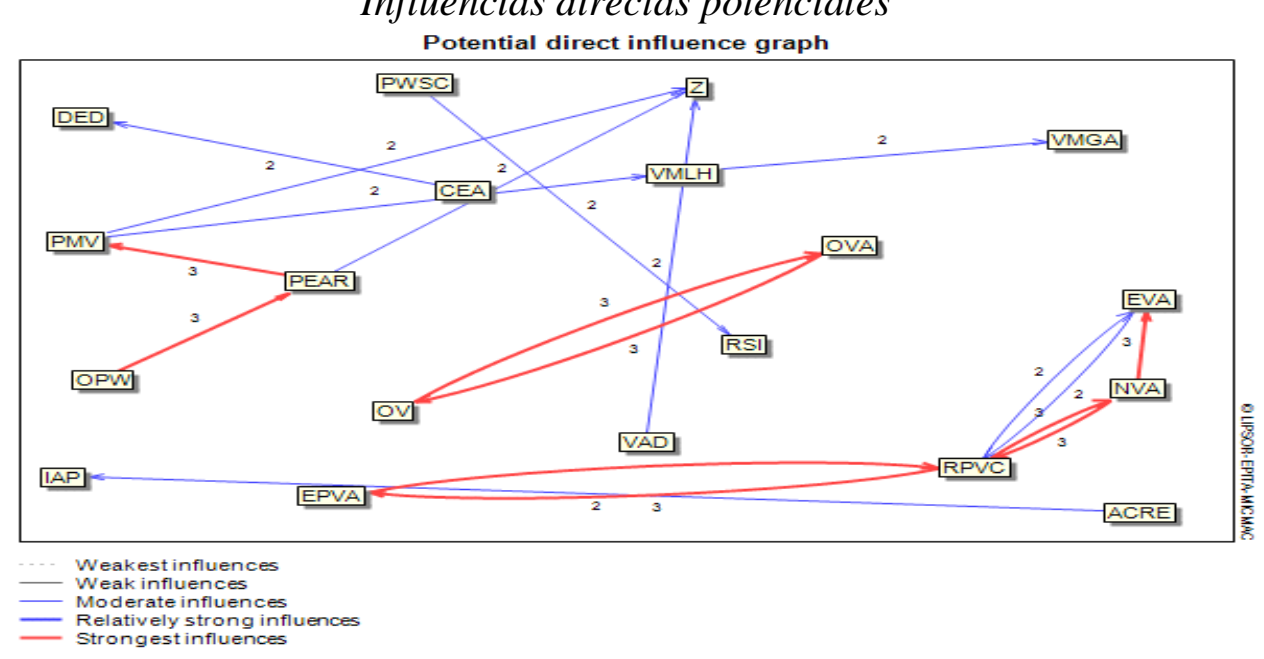


Determinada las variables que influyen según el análisis estructural se continúa con la elaboración de los espacios morfológicos (hipótesis) para el comportamiento del mercado chino en la etapa post COVID del sector turístico cubano. De los 625 escenarios posibles la aplicación sólo considera 50 escenarios definidos como los más probables (ver figura 4), de ellos se retuvieron para el posterior estudio los cuatro primeros escenarios.

Figura 4

\section{Lista de escenarios generados}
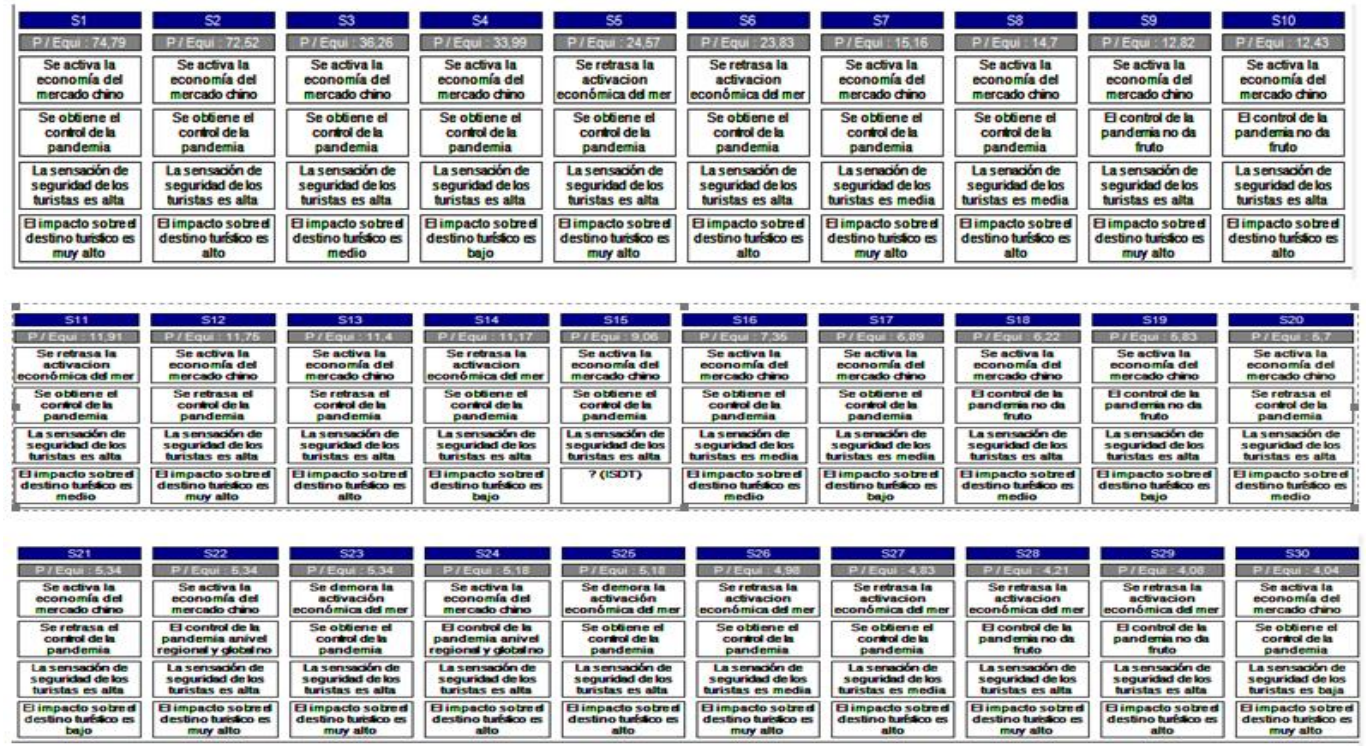

Para precisar el escenario de prioridad o futurible se emplean los cuatro escenarios seleccionados a través del análisis morfológico y a estos se les aplica el método de Sistema de Matrices de Impactos Cruzados con el uso del software Smic-Prob-Expert. En este software se procesaron tres hipótesis a partir de la variable recuperación de la actividad turística y afluencia del mercado turístico chino. El análisis de los datos procesador por el software arrojó que la hipótesis 3 (Con una recuperación en la actividad turística a partir de finales del mes de octubre es posible una reducción de la afluencia turistas chinos del 30\%) es la más probable por el $100 \%$ de los encuestados como se visualiza en la figura 5 .

\section{Figura 5}

Histograma de probabilidades simples de todos los expertos

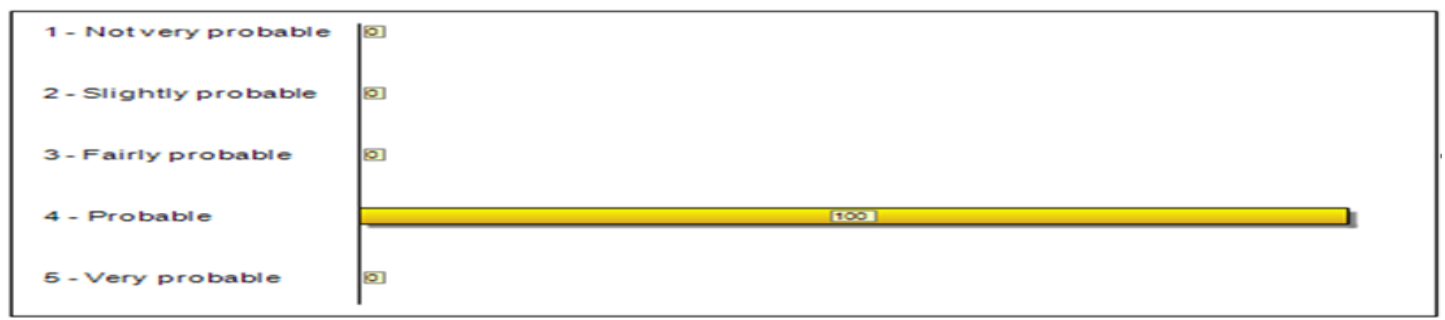


Con la ayuda del método multicriterio a través del software MULTIPOL y a partir de criterios de expertos y especialistas sobre la investigación de mercados en el sector turístico cubano se determinan las políticas y acciones para atraer el mercado chino utilizando para ello los cuatros escenarios seleccionados a través del análisis morfológico. En la figura 6 se observa que en el escenario 1 el criterio que más valor tienes es el impacto del sector turístico, mientras que en los otros escenarios el criterio que más predomina es el económico según los elementos aportados por expertos y especialistas.

\section{Figura 6}

Mapa de perfil de escenarios

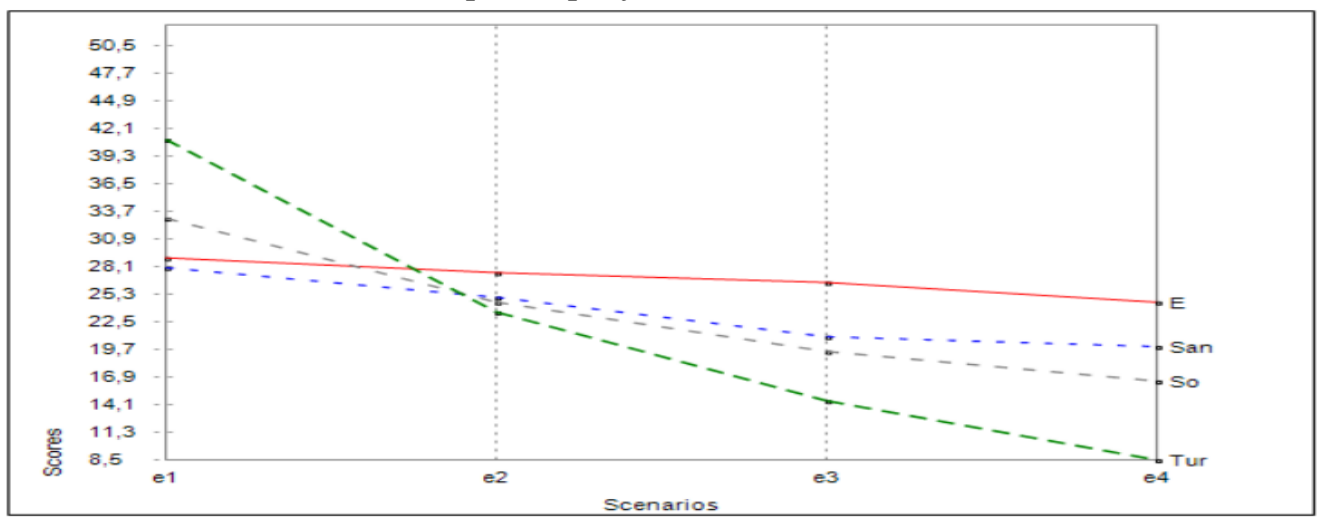

Otro resultado fue el mapa de perfil de las políticas con respecto a las acciones que se deben acometer (ver figura 7), en ella se visualiza los valores más altos que se corresponden con la acción 2 de controlar la pandemia en el mercado emisor chino y la acción 3 de mejorar la seguridad en destino turístico cubano.

Figura 7

Mapa de perfiles de las políticas con respecto a las acciones

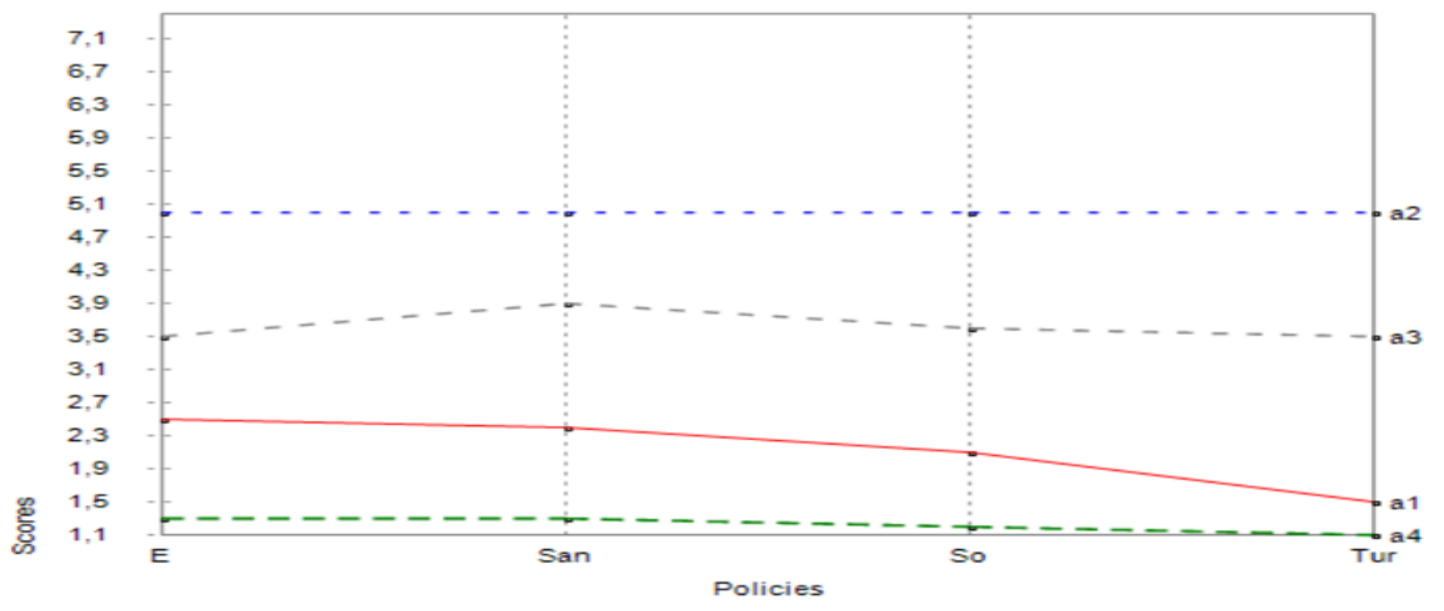




\section{Conclusiones}

Con la realización de la presente investigación se pudo concluir:

- Los resultados obtenidos con la aplicación de los instrumentos y métodos permiten conocer las potencialidades y restricciones fundamentales del comportamiento del mercado emisor chino para la etapa post COVID del sector turístico cubano.

- Todas las acciones que se propongan deben disminuir las debilidades y potenciar las oportunidades para atraer al turista chino.

- El análisis de prospectiva realizado pudo determinar las variables que más influyen en el comportamiento del mercador emisor chino.

- Se pudo determinar cuatro escenarios probables del mercado emisor chino para la etapa post COVID del sector turístico cubano.

- A partir de las variables claves, se acuerda una hipótesis probable, para las cuales se conciernan cuatro políticas y dos acciones a cumplimentar por los tomadores de decisión para lograr atraer al mercado emisor chino.

\section{Referencias Bibliográficas}

Astigarraga, E. (2010). Estrategia Empresarial Prospectiva. Madrid: Universidad de Deusto.

Cely, A. (1999). Metodología de los escenarios para estudios Bogotá: Revista Ingeniería e Investigación prospectivos (Vol. 44). (U. N. Colombia, Ed.)

Cornejo, J. L., Chávez, R. M. \& Espinoza, R. (2019). Prospectiva del turismo de naturaleza en la costa de Jalisco. Investigaciones Turísticas (17), pp. 189-212. http://dx.doi.org/10.14198/ INTURI2019.17.09.

Delgado, M. D. (2015). Exploring Prospective Structural Analysis to Assess the Relevance of Rural Territorial Development in Spain and Nicaragua. Bogota: Cuad. Des. Rural. Universidad Javeriana.

Godet, M (1993). De la anticipación a la acción. Manual de Prospectiva y Estrategia. Marcombo. España.

Godet, M. (2000). La caja de herramientas de la prospectiva estratégica. Paris, Francia: Gerpa, Electricité de France, Mission Prospective.

Hernández, Y. \& Machado, E.L. (2010). Estrategia de posicionamiento del destino Villa Clara en el mercado chino. Revista Teoría y Praxis, No 7, pág.55-77. ISSN:18701582 
Lojo, A. (2016). Turismo Chino Emisor: estado de la cuestión y agenda. Boletín de la Asociación de Geógrafos Españoles, N. ${ }^{\circ}$ 72, pág. 471-500. ISSN: 0212-9426. DOI:10.21138/bage. 2348

Mao, I. \& Zhang, H.Q. (2014): Structural Relationships among Destination Preference, Satisfaction and Loyalty in Chinese Tourists to Australia. International Journal of Tourism Research, vol. 16, 201-208.

Munera, N.J. (2016). Revisión de los métodos de prospectiva tecnológica para la adaptación de un modelo prospectivo aplicable al programa "Medellín Espacial" - Ruta N. (Trabajo de grado para optar al título de Magister en Gerencia de Proyectos. Escuela de Administración). Universidad EAFIT. Medellín. Colombia.p.78.

Rincón, S. \& Mujica, N (2004). Estudio prospectivo de la gestión tecnológica en las empresas del sector metalmecánico del estado Zulia. Revista Venezolana de Gerencia, vol. 9, núm. 26, abril-junio, pp. 289-314. Venezuela.

Sánchez, G. (2003). Técnicas participativas para la planeación: Procesos breves de intervención. México: Fundación ICA.

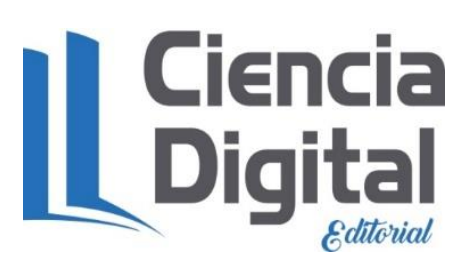




\section{EPDigital}

El artículo que se publica es de exclusiva responsabilidad de los autores y no necesariamente reflejan el pensamiento de la Revista Explorador Digital.

\section{\Cigital}

El artículo queda en propiedad de la revista y, por tanto, su publicación parcial y/o total en otro medio tiene que ser autorizado por el director de la Revista Explorador Digital.
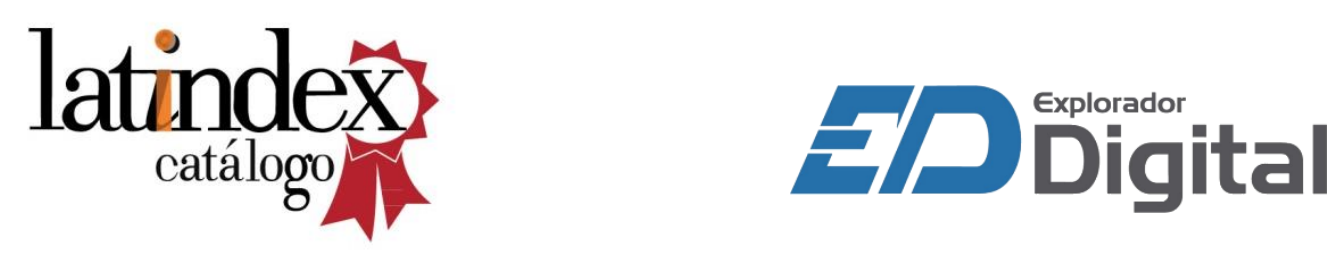

Indexaciones

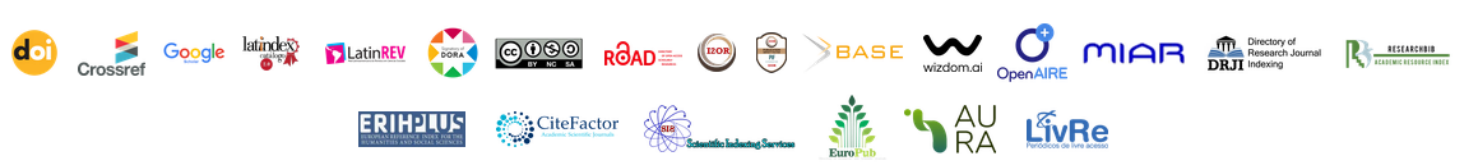

